\title{
Reproductive outcomes in recurrent pregnancy loss associated with a parental carrier of chromosome abnormalities or polymorphisms
}

\author{
Y. Dong ${ }^{1}$, L.L. Li ${ }^{2}$, R.X. Wang ${ }^{1}$, X.W. Yu ${ }^{1}$, X. Yun ${ }^{2}$ and R.Z. Liu ${ }^{1}$ \\ ${ }^{1}$ Center for Reproductive Medicine, Center for Prenatal Diagnosis, \\ First Hospital, Jilin University, Changchun, China \\ ${ }^{2}$ Department of Cell Biology, Norman Bethune College of Medicine, \\ Jilin University, Changchun, China \\ Corresponding author: R.Z. Liu \\ E-mail:1rz410@126.com
}

Genet. Mol. Res. 13 (2): 2849-2856 (2014)

Received May 8, 2013

Accepted September 20, 2013

Published January 17, 2014

DOI http://dx.doi.org/10.4238/2014.January.17.4

\begin{abstract}
The subsequent reproductive outcomes in couples with a history of recurrent pregnancy loss (RPL) associated with chromosome abnormalities or polymorphisms are generally not reported in China. Many RPL carrier couples have decided not to have children. The present study recorded the subsequent delivery, miscarriage, and unpregnancy outcomes of 113 RPL carrier couples and 226 non-carrier couples, and compared differences in reproductive outcomes between couples with different types of chromosome abnormalities or polymorphisms and chromosome normal couples. Our results showed that couples with RPL associated with parental chromosome abnormalities or polymorphisms did not have significantly lower live birth rates than non-carrier couples in China. These results suggest the current guidance given to Chinese RPL couples.
\end{abstract}

Key words: Chromosome rearrangement; Chromosome abnormality; Recurrent pregnancy loss; Polymorphism 


\section{INTRODUCTION}

Recurrent pregnancy loss (RPL), generally defined as two or more pregnancy losses before 20 weeks of gestation (Stephenson and Kutteh, 2007), occurs in 2-5\% of all couples trying to conceive (Christiansen et al., 2005). Several factors are associated with RPL, such as congenital or structural uterine anomalies, endocrine dysfunction, autoimmune disorders, infectious diseases, and blood coagulation factors, among others. However, the etiology of nearly $50 \%$ of such cases is unknown (Stephenson, 1996). The presence of chromosome abnormalities in a parental carrier is one of the most reliable etiologies, and balanced chromosomal rearrangements have been observed in couples with RPL (De Braekeleeret and Dao, 1990). The effectiveness of pre-implantation genetic diagnosis (PGD) remains elusive (Ozawa et al., 2008). Sugiura-Ogasawara et al. (2004) reported that PGD for RPL couples is not necessary. However, Fischer et al. (2010) proposed that individuals with chromosome translocations who have experienced RPL would benefit from PGD by realizing a significant reduction in loss rate and improvement in the rate of successful pregnancies.

To our knowledge, little information is available on RPL prognosis the in such carrier couples through natural means of pregnancy in China. In the present study, we determined the incidence and types of chromosome abnormalities or polymorphisms in RPL couples. More importantly, we aimed to investigate long-term reproductive outcomes in RPL couples, and to compare such outcomes in carriers to those of non-carriers. This information should prove to be useful for counseling naturally pregnant carrier couples with a history of RPL about their chances of having a healthy child and their risk of miscarriage or unpregnancy.

\section{MATERIAL AND METHODS}

\section{Patients}

Couples with RPL $(\mathrm{N}=2720)$ who sought chromosome karyotype analysis from the Centre for Reproductive Medicine, the First, Second, and Third Hospital of Jilin University, Changchun, China were recruited for this study between June 2007 and November 2011. Each couple was also subjected to other examinations to investigate the potential causes of RPL, such as hysterosalpingography, luteal phase assessment, immunological tests, infection factors tests, clotting inspection, and blood tests for diabetes mellitus, hyperthyroidism, etc. The carrier couples were treated for all the above possible causes of RPL except chromosome abnormalities in the same manner as non-carrier couples. Before inclusion in our study, objective evidence of past pregnancies was required, such as a positive human chorionic gonadotropin (hCG) test and ultrasound-based reports of a gestational sac. When one partner was found to carry chromosome abnormalities or polymorphisms, we identified the couple as a carrier couple and the patient as a carrier. In view of the large number of RPL couples, we selected a random subset of two non-carrier couples per carrier couple by identifying the non-carrier couples who were tested immediately before and after their matched carrier couples $(\mathrm{N}=226)$. This matching was designed with the aim of obtaining a sample that was balanced over time. Data collection by means of telephone interview focused on the reproductive outcomes of couples followed for at least 12 months after chromosome analysis. Of the RPL carrier couples with chromosome abnormalities or polymorphisms, 18 of 
the carriers' family members were recalled back to be interviewed about their reproductive histories and underwent with chromosome karyotype analyses. All participants provided written informed consent, and the study was approved by the Ethics Committee of Jilin University, Changchun, China.

\section{Karyotype analysis}

Karyotype analysis was performed on all couples $(\mathrm{N}=2720)$ and family members of the 18 carriers. Briefly, $0.8 \mathrm{~mL}$ peripheral blood lymphocytes were cultured in commercial lymphocyte culture medium (Yishengjun; BaiDi Bio-Technology, Guangzhou, China) at $37^{\circ} \mathrm{C}$ for $72 \mathrm{~h}$, followed by the addition of $50 \mu \mathrm{g} / \mathrm{mL}$ colchicine (Yishengjun; BaiDi Bio-Technology) to arrest mitosis for $1 \mathrm{~h}$ before culture termination. Harvesting of peripheral blood lymphocytes was performed by hypotension, fixation, trypsinization, and Giemsa banding (GTG-banding) at 350-400 bands level. Detailed experimental procedures were performed as previously described (Dong et al., 2012).

Selective banding studies, such as C-banding, were performed for heterochromatin polymorphisms, and NOR-banding was performed for satellites/stalks variations. At least 20 metaphases were analyzed for each individual. Mosaicisms were counted for at least 50 metaphases. Chromosome abnormalities and polymorphisms were reported according to the International System for Human Cytogenetic Nomenclature (ISCN, 2009) (Shaffer and Slovak, 2009). It is worth noting that the pericentric inversion of chromosome 9 [inv (9)] was also considered to be as a polymorphism (Hong et al., 2011) in this study.

\section{Statistical analysis}

Statistical analysis was performed with the SPSS $^{\circledR}$ version 17.0 statistical package (SPSS Inc., Chicago, IL, USA) for Windows. We tested the differences between carrier ( $\mathrm{N}=$ $113)$ and non-carrier couples $(\mathrm{N}=226)$ who participated in our study with the Mann-Whitney $\mathrm{U}$ test for nonparametric continuous variables, and the chi-squared and Fisher's exact tests for categorical variables. A P value $<0.05$ was considered to be statistically significant and all $\mathrm{P}$ values were two-sided.

\section{RESULTS}

The data set consisted of 2720 couples (mean age of 30.7 years, range 19-45 years) with a history of RPL. Among the 2720 couples, there were 137 carriers $(5.04 \%, 137 / 2720)$ who were divided into two types: 61 cases of chromosome abnormalities $(2.24 \%$, male $=27$, female $=34)$, including 35 cases of reciprocal translocations $(1.29 \%)$, 10 cases of Robertsonian translocations $(0.37 \%)$, seven cases of inversions $(0.26 \%)$, five cases of sex chromosome aneuploidies $(0.18 \%)$, three cases of mosaicisms $(0.11 \%$, female), and one case of complex translocation $(0.04 \%$, male), and 76 cases of chromosome polymorphisms $(2.80 \%$, male $=44$, female $=32$ ), including 55 cases of $\mathrm{pstk} \pm / \mathrm{qh} \pm$ pss $(2.02 \%)$ and 21 cases of $\operatorname{inv}(9)(0.77 \%)$ (Table 1). There was no significant difference in the frequency of males and females with different types of chromosome abnormalities or polymorphisms $(\mathrm{P}>0.05)$; however, Robertsonian translocations were more frequently observed in females (Table 1). 
Table 1. Details in parental carriers and polymorphisms.

\begin{tabular}{|c|c|c|c|c|}
\hline & Types of anomaly & Male & Female & Total $^{\mathrm{a}, \mathrm{b}}$ \\
\hline \multirow[t]{6}{*}{ Abnormalities } & Reciprocal translocation & 19 & 16 & $35(25.55 \%)$ \\
\hline & Robertsonian translocation & 2 & 8 & $10(7.30 \%)$ \\
\hline & Inversion & 2 & 5 & $7(5.11 \%)$ \\
\hline & Sex chromosome aneuploidies & 3 & 2 & $5(3.65 \%)$ \\
\hline & Mosaicism & 0 & 3 & $3(2.19 \%)$ \\
\hline & Complex translocation & 1 & 0 & $1(0.73 \%)$ \\
\hline \multirow[t]{2}{*}{ Polymorphisms } & $\mathrm{pstk} \pm / \mathrm{qh} \pm / \mathrm{pss}$ & 32 & 23 & $55(40.15 \%)$ \\
\hline & Inversion(9) & 12 & 9 & $21(15.33 \%)$ \\
\hline Total & & 71 & 66 & $137(100 \%)$ \\
\hline
\end{tabular}

${ }^{a}$ Numbers in parenthesis are percentages in all the chromosome abnormalities. ${ }^{b}$ Fisher's exact test. $\mathrm{P}>0.05$ the frequency of different types of chromosome abnormalities or polymorphisms in males and females was not significant difference.

Among the 137 carrier couples, 113 couples participated in our study and they were divided into five groups: reciprocal translocation, Robertsonian translocation, inversion, inversion (9), and $\mathrm{pstk} \pm / \mathrm{qh} \pm / \mathrm{pss}$. The reasons for other 24 carrier couples not participating in this study included refusal to participate, non-response, and wrong telephone number. We also invited 226 chromosome normal couples to participate in our study as a control group.

As shown in Table 2, there was no significant difference between the normal chromosome group and the other five groups (reciprocal translocation, Robertsonian translocation, inversion, inversion(9), and $\mathrm{pstk} \pm / \mathrm{qh} \pm / \mathrm{pss}$ ) with respect to maternal age and previous numbers of RPL carriers $(\mathrm{P}=0.388 ; \mathrm{P}=0.564)$. In the analyses of the subsequent reproductive outcomes of the 339 participant couples, there were no significant difference between the normal chromosome group and the other five chromosomal abnormality or polymorphism groups with respect to delivery, miscarriage, and unpregnancy rates $(\mathrm{P}>$ 0.05). There was also no significant difference in the reproductive outcome between males and females with chromosome abnormalities or polymorphisms. It is worth noting that among the 113 carrier couples, five couples decided not to conceive in order to avoid RPL.

Table 2. Results of subsequent reproductive outcomes in 113 carrier couples and 226 non-carrier couples.

\begin{tabular}{|c|c|c|c|c|c|c|}
\hline & Normal & Reciprocal translocation & Robertsonian translocation & Inversion & Inversion(9) & $\mathrm{pstk} \pm / \mathrm{qh} \pm / \mathrm{pss}$ \\
\hline No. of couples & 226 & 32 & 9 & 5 & 17 & 50 \\
\hline Maternal age $\mathrm{a}^{\mathrm{a}, \mathrm{b}}$ & $30.3 \pm 4.6$ & $31.6 \pm 6.1$ & $32.7 \pm 5.5$ & $29.8 \pm 5.4$ & $30.5 \pm 5.4$ & $31.3 \pm 5.0$ \\
\hline Range & $22-45$ & $19-44$ & $25-40$ & 25-39 & $22-40$ & $21-42$ \\
\hline No. of past RPL ${ }^{b}$ & $2.7 \pm 1.3$ & $2.6 \pm 0.9$ & $2.4 \pm 0.7$ & $2.6 \pm 0.9$ & $2.8 \pm 1.1$ & $2.9 \pm 1.5$ \\
\hline Range & $2-6$ & $2-6$ & $2-4$ & $2-4$ & $2-6$ & $2-11$ \\
\hline Delivery $(\%)^{\mathrm{c}}$ & $128(56.6 \%)$ & $16(50.0 \%)$ & $3(33.3 \%)$ & $2(40.0 \%)$ & $9(52.9 \%)$ & $28(56.0 \%)$ \\
\hline P value ${ }^{c}$ & & 0.569 & 0.189 & 0.656 & 0.804 & 1.000 \\
\hline Miscarriage $(\%)^{\mathrm{c}}$ & $27(11.9 \%)$ & $5(15.6 \%)$ & $1(11.1 \%)$ & $1(20.0 \%)$ & $3(17.6 \%)$ & $5(10.0 \%)$ \\
\hline $\mathrm{P}_{\text {value }}$ & & 0.567 & 1.000 & 0.479 & 0.449 & 0.811 \\
\hline Unpregnancy $(\%)^{\mathrm{c}}$ & $71(31.4 \%)$ & $11(34.4 \%)$ & $5(55.6 \%)$ & $2(40.0 \%)$ & $5(29.4 \%)$ & $17(34.0 \%)$ \\
\hline$P$ value ${ }^{c}$ & & 0.839 & 0.153 & 0.652 & 1.000 & 0.739 \\
\hline
\end{tabular}

${ }^{a}$ Data are reported as means \pm SD. Numbers in parenthesis are percentages. ${ }^{b}$ Mann-Whitney $U$ test. $P>0.05$. There was no significant difference in maternal age and previous numbers of RPL carriers $(\mathrm{P}=0.388 ; \mathrm{P}=0.564)$ between the chromosome normal group and the other five groups [Reciprocal translocation/Robertsonian translocation/ Inversion/Inversion(9)/ $\mathrm{pstk} \pm / \mathrm{qh} \pm / \mathrm{pss}]$. 'Fisher's exact test. $\mathrm{P}>0.05$. There was no significant difference in delivery, miscarriage, unpregnancy rate between the chromosome normal group and the other five groups. 
According to the pedigree analysis of the 18 carriers, the chromosome abnormalities were inherited from the mothers in 10 cases, from the fathers in seven cases, and in one case from his mother and de novo simultaneously. None of the carriers' fathers, mothers, or even siblings with the same chromosomal abnormalities and polymorphisms had the same RPL history, and instead had normal reproductive histories, with the exception of family number 13. The reproductive outcomes of the 18 carriers are shown in Table 3.

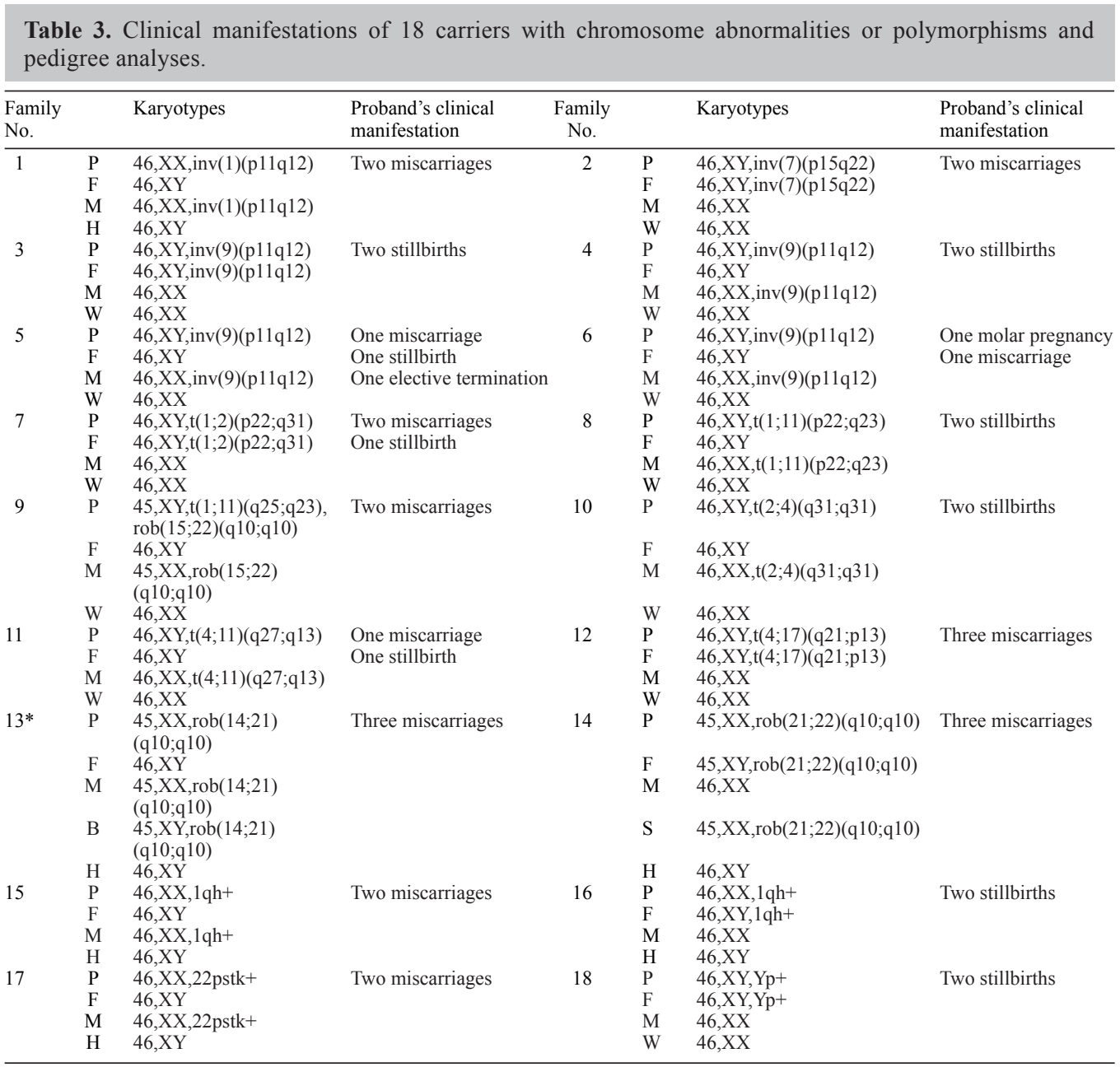

$\mathrm{P}=$ proband $\mathrm{F}$ = father; $\mathrm{M}=$ mother; $\mathrm{B}$ = brother; $\mathrm{S}=$ sister; $\mathrm{W}=$ wife; $\mathrm{H}=$ husband; Family No.13*: the carrier's mother had two miscarriages, other parents had no adverse reproductive history. Family No. 1, 3, 5, 12, 15, 16, 17, 18: the carrier couples had born normal phenotype children. Family No. 2, 4, 7, 9, 10, 14: the carrier couples had secondary infertility. Family No. 6, 8, 9, 11: the carrier couples had not been participated in this study and their reproductive outcomes were not known.

\section{DISCUSSION}

In the present study, the prevalence of chromosome abnormalities or polymorphisms 
in RPL couples was 5.04\%. Previous studies have reported the incidence of these abnormalities and polymorphisms at 3-6\% (Tharapel et al., 1985; De Braekeleeret and Dao, 1990; Clifford et al., 1994; Franssen et al., 2005). Among the 2720 RPL couples, 2.24\% of carriers had chromosome abnormalities and $2.80 \%$ had polymorphisms. Previous studies have reported that $2.5-7.8 \%$ of RPL couples were carriers of structural chromosome rearrangements (Stephenson, 1996; Goddijn et al., 2004; Sugiura-Ogasawara et al., 2008), whereas only $0.2 \%$ of the general reproductive population has chromosomal rearrangements (Grimm, 2010). The incidence of polymorphic variants was $8.9 \%$ in couples with two or more consecutive gestational losses (Campanho et al., 2011). These differences among studies may be related to the sample size and to different criteria.

The risk of miscarriage or unpregnancy depends on the chromosome number and size of the chromosomal segment involved (Boue and Gallano, 1984; Ogilvie and Scriven, 2004). Thus, chromosome rearrangement carriers, although expressing normal phenotypes, may nonetheless produce unbalanced gametes through unequal meiotic segregation or recombination during gametogenesis (Ogilvie and Scriven, 2004). With respect to reciprocal translocation, carriers have a significantly increased risk ( $50 \%$ or more) of chromosomal imbalance (partial chromosomal duplication/deficiency) during gametogenesis due to unequal meiotic segregation (Celep et al., 2006). However, in our cohort, the delivery rate of reciprocal translocation carrier couples was similar to that of non-carrier couples (50vs $56.6 \%, \mathrm{P}=0.569$ ), and the risk of miscarriage was far lower than $50 \%$. In other words, the actual rate for chromosomal abnormality couples of having a healthy child was far higher than the theoretical value. The delivery rate of the other four groups (Robertsonian translocation, inversion, inversion(9), and $\mathrm{pstk} \pm / \mathrm{qh} \pm / \mathrm{pss}$ ) was $33.3,40.0,52.9$, and $56.0 \%$ respectively, which was also not significantly lower than that of the non-carrier group.

Additionally, analysis of the subsequent reproductive outcomes of the 339 participant couples revealed no significant differences between the normal chromosome group and the other five chromosomal abnormality or polymorphism groups with respect to the delivery, miscarriage, and unpregnancy rates $(\mathrm{P}>0.05)$. These data indicate that the probabilities of chromosomal abnormality or polymorphic carriers of having healthy children and their risk of miscarriage or unpregnancy were similar to those of non-carrier couples. There were also no statistically significant differences in the subsequent delivery, miscarriage, and unpregnancy rates between male and female carriers with different types of chromosome abnormalities or polymorphisms.

To confirm the effect of chromosome abnormalities or polymorphisms on RPL couples, we conducted further investigations based on pedigree analyses. Although 17 of the carriers' abnormal karyotypes were inherited from one of their parents, their parents did not show the same adverse reproductive history, except for family number 13 . This suggests that the abnormal karyotypes may not be the only cause of the RPL. Therefore, such carrier couples should be examined for other etiologies, such as antiphospholipid antibodies, uterine anomalies, and immunological abnormalities, among others. Because different factors may interact, evaluation and management of other factors associated with RPL may improve the subsequent live birth rate in carriers. However, we could not eliminate the possibility that some miscarriages might have occurred very early in the pregnancy, shortly after fertilization, and thus mothers of carriers were unaware of the pregnancy. The fact that 17 parents without a history of reproductive disadvantage is neither a coincidence nor neglect of the carriers' mothers, 
which illustrates that couples with chromosome abnormalities can still birth healthy children after having had a few adverse reproductive events or even without RPL.

Unfortunately the 58 carrier couples who had given birth to healthy children did not receive prenatal diagnoses. We could not recognize the children's karyotypes, but only normal phenotypes. In addition, between June 2007 to November 2011 in the present study, 40 of the 113 carrier couples and 71 of the 226 non-carrier couples were cases of unpregnancy. It is possible that over a longer follow-up period, more RPL couples might have conceived and more accurate information could have been obtained on reproductive outcomes.

In conclusion, the chance of having a healthy child and the risk of miscarriage and unpregnancy were similar between the carrier and non-carrier couples. This finding could suggest that RPL carrier couples and couples who decided not to conceive should nonetheless prepare for pregnancy. This information makes a case for changing the current guidance given to these Chinese RPL couples.

\section{ACKNOWLEDGMENTS}

We thank all of the couples and their parents for participating in this study. We also thank the staff of the Cytogenetic Laboratory for their excellent work. This research was kindly supported by funds from the National Population and Family Planning Commission of China (\#2011-GJKJS-07).

\section{REFERENCES}

Boue A and Gallano P (1984). A collaborative study of the segregation of inherited chromosome structural rearrangements in 1356 prenatal diagnoses. Prenat. Diagn. 4 Spec. No: 45-67.

Campanho CL, Heinrich JK, Couto E and Barini R (2011). Subfertility phenotype, chromosome polymorphism and conception failures. Rev. Bras. Ginecol. Obstet. 33: 246-251.

Celep F, Karaguzel A, Ozeren M and Bozkaya H (2006). The frequency of chromosomal abnormalities in patients with reproductive failure. Eur. J. Obstet. Gynecol. Reprod. Biol. 127: 106-109.

Christiansen OB, Nybo Andersen AM, Bosch E, Daya S, et al. (2005). Evidence-based investigations and treatments of recurrent pregnancy loss. Fertil. Steril. 83: 821-839.

Clifford K, Rai R, Watson H and Regan L (1994). An informative protocol for the investigation of recurrent miscarriage: preliminary experience of 500 consecutive cases. Hum. Reprod. 9: 1328-1332.

De Braekeleer M and Dao TN (1990). Cytogenetic studies in couples experiencing repeated pregnancy losses. Hum. Reprod. 5: 519-528.

Dong Y, Du RC, Jiang YT, Wu J, et al. (2012). Impact of chromosomal translocations on male infertility, semen quality, testicular volume and reproductive hormone levels. J. Int. Med. Res. 40: 2274-2283.

Fischer J, Colls P, Escudero T and Munne S (2010). Preimplantation genetic diagnosis (PGD) improves pregnancy outcome for translocation carriers with a history of recurrent losses. Fertil. Steril. 94: 283-289.

Franssen MT, Korevaar JC, Leschot NJ, Bossuyt PM, et al. (2005). Selective chromosome analysis in couples with two or more miscarriages: case-control study. BMJ 331: 137-141.

Goddijn M, Joosten JH, Knegt AC, van dF, et al. (2004). Clinical relevance of diagnosing structural chromosome abnormalities in couples with repeated miscarriage. Hum. Reprod. 19: 1013-1017.

Grimm TZK (2010). Genetic Counseling and Prenatal Diagnosis. In: Vogel and Motulsky's Human Genetics. 4th ed. (Speicher MR, Motulsky AG and Antonarakis SE, eds.). Springer, Berlin Heidelberg, 845.

Hong Y, Zhou YW, Tao J, Wang SX, et al. (2011). Do polymorphic variants of chromosomes affect the outcome of in vitro fertilization and embryo transfer treatment? Hum. Reprod. 26: 933-940.

Ogilvie CM and Scriven PN (2004). Preimplantation genetic diagnosis (PGD) for reciprocal translocations. Prenat. Diagn. 24: 553-555.

Ozawa N, Maruyama T, Nagashima T, Ono M, et al. (2008). Pregnancy outcomes of reciprocal translocation carriers who 
have a history of repeated pregnancy loss. Fertil. Steril. 90: 1301-1304.

Shaffer LG and Slovak ML (2009). International Software Consulting Network (ISCN). In: An International System for Human Cytogenetic Nomenclature (S. Karger, ed.). Basel, 53-87.

Stephenson M and Kutteh W (2007). Evaluation and management of recurrent early pregnancy loss. Clin. Obstet. Gynecol. 50: $132-145$.

Stephenson MD (1996). Frequency of factors associated with habitual abortion in 197 couples. Fertil. Steril. 66: 24-29.

Sugiura-Ogasawara M, Ozaki Y, Sato T, Suzumori N, et al. (2004). Poor prognosis of recurrent aborters with either maternal or paternal reciprocal translocations. Fertil. Steril. 81: 367-373.

Sugiura-Ogasawara M, Aoki K, Fujii T, Fujita T, et al. (2008). Subsequent pregnancy outcomes in recurrent miscarriage patients with a paternal or maternal carrier of a structural chromosome rearrangement. J. Hum. Genet. 53: 622-628.

Tharapel AT, Tharapel SA and Bannerman RM (1985). Recurrent pregnancy losses and parental chromosome abnormalities: a review. Br. J. Obstet. Gynaecol. 92: 899-914. 